\title{
Ten novel insertion/deletion variants in MECP2 identified in Japanese patients with Rett syndrome
}

\author{
Eri Takeshita', Aritoshi lida $\mathbb{D}^{2}$, Chihiro Abe-Hatano ${ }^{3}$, Eiji Nakagawa', Masayuki Sasaki', Ken Inoue ${ }^{3}$ and Yu-ichi Goto ${ }^{3,4}$
}

\begin{abstract}
Rett syndrome (RTT) is an X-linked progressive and severe neurological disorder caused by mutations in the gene encoding methyl CpG binding protein 2 (MECP2). Among the 49 typical RTT patients examined, we identified 10 novel and eight known insertion/deletion variants, and 31 known pathogenic variants in MECP2. The pathogenic variants presented here should be a useful resource for examining the correlation between the genotypes and phenotypes of RTT.
\end{abstract}

Rett syndrome (RTT) is a progressive neurodevelopmental disorder that affects brain development and function in females, with a prevalence of one in 10,000 worldwide $^{1}$. Typical RTT is caused by mutations in the gene encoding methyl-CpG binding protein $2(M E C P 2)^{2}$. A database of a large collection of $M E C P 2$ variants was established in 2002 (RettBASE: http://mecp2.chw.edu.au/ index.shtml $)^{3}$. To date, associations between clinical phenotypes and related genetic variants for $M E C P 2$ as well as other RTT-associated genes, including CDKL5 and FOXG1, are available.

Here, we report a total of 49 RTT patients with 10 novel insertion/deletion variants, eight known insertion/deletion variants and 31 known pathological variants.

All patients were diagnosed with typical RTT by Japanese child neurology experts according to the international diagnostic criteria for RTT. Clinical information and samples from the patient and parents were obtained with written informed consent. The study was approved by the ethical committee of NCNP. Genomic DNA was extracted from peripheral blood using a standard protocol. We first used the MLPA method (MRC-Holland, DL Amsterdam, The Netherlands) to identify the structural abnormalities in the MECP2 locus. In the patients excluded for structural

\footnotetext{
Correspondence: Yu-ichi Goto (goto@ncnp.go.jp)

'Department of Child Neurology, National Center Hospital, National Center of Neurology and Psychiatry (NCNP), Kodaira, Tokyo 187-8551, Japan ${ }^{2}$ Department of Clinical Genome Analysis, Medical Genome Center, NCNP, Kodaira, Tokyo 187-8551, Japan

Full list of author information is available at the end of the article.

These authors contributed equally: Eri Takeshita, Aritoshi lida
}

abnormalities, we amplified all coding exons of $M E C P 2$ and their exon-intron boundaries by PCR and directly sequenced the PCR products using the Applied Biosystems 3730 DNA analyzer (Thermo Fisher, USA).

The insertion/deletion mutations were detected in 18 (36.7\%) of 49 patients with RTT (Table 1). Among the 18 patients, 10 (55.6\%) were considered novel by a comparison of our data with the known insertions/deletions deposited in the public databases, including RettBASE, gnomAD, Human Genome Mutation Database Professional 2019.2 and ClinVar. Representative data of pedigrees and sequences of the recombination breakpoints from three families are shown in Fig. 1. Patient 470 showed the insertion/ deletion variant at c.1158_1258delinsCCGAGGGTGGCT CC. Patient 488 showed the deletion at c.1168 ${ }_{-}^{*} 539$ del. Patient 587 showed the insertion/deletion at c.1367_791 delinsCGC. Five (Patients 187, 470, 488, 559, and 587) had lost only exon 4 . In addition, Patient 269 has a complex rearrangement with a $2609 \mathrm{bp}$ deletion, including exon 3 and flanking introns, accompanied by two nucleotide substitution and a 25 bp deletion in exon4, c. [27-1707_c.378206del; 1159_1160CC>AG; 1164_1188del], occurred in cis. The other five showed intragenic deletion involving exon 4 in the MECP2 locus. Hardwick et al. (2007) reported that 12 out of 21 patients $(57 \%)^{4}$ experienced breakpoints within the "deletion-prone region (DPR)", which is characterized by short direct repeat elements and is also known as the hotspot for the smaller deletions ${ }^{5,6}$. In this study, the breakpoints in seven novel insertion/deletion variants (7/10: $70 \%$ ) were located within the DPR. In the breakpoints of 


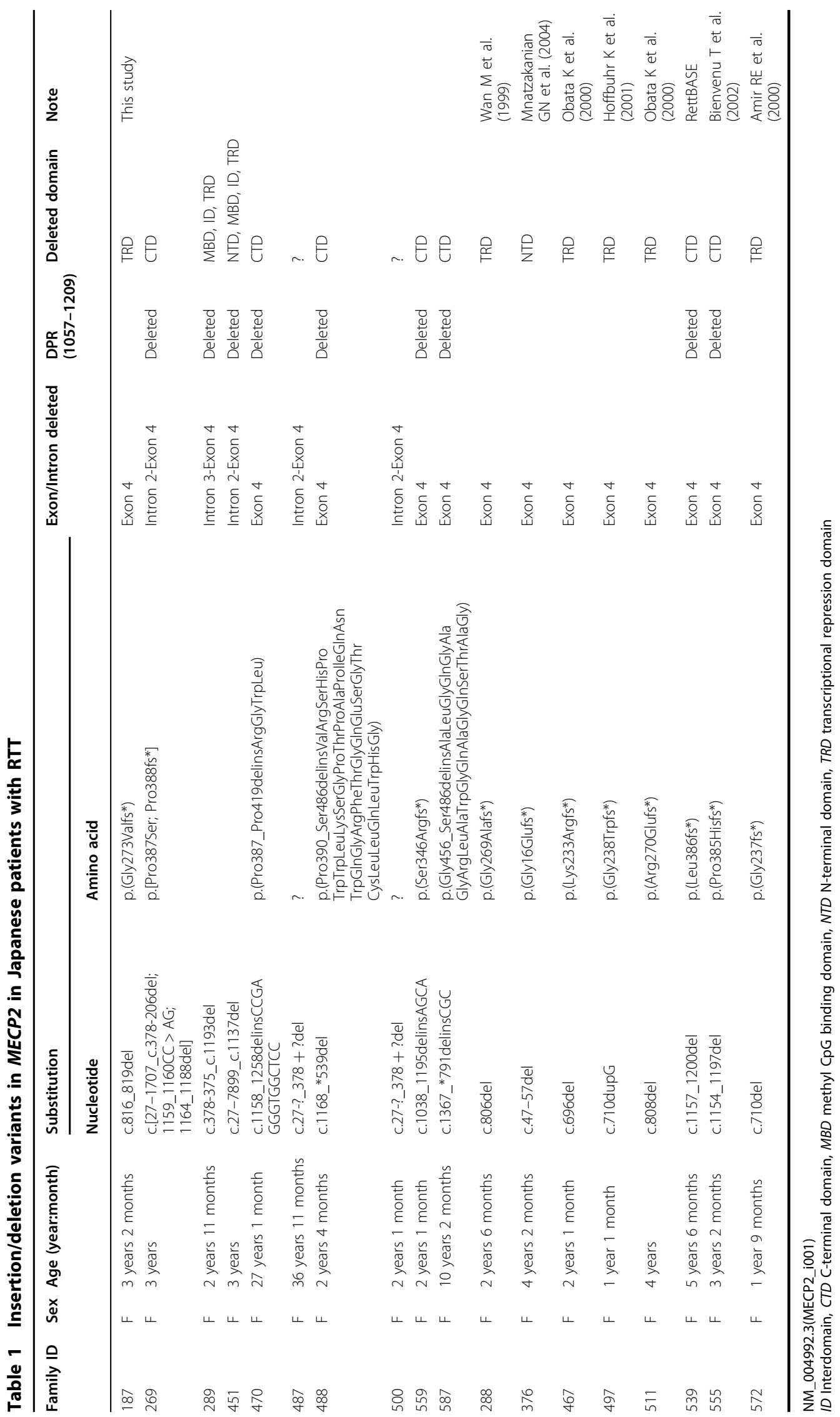




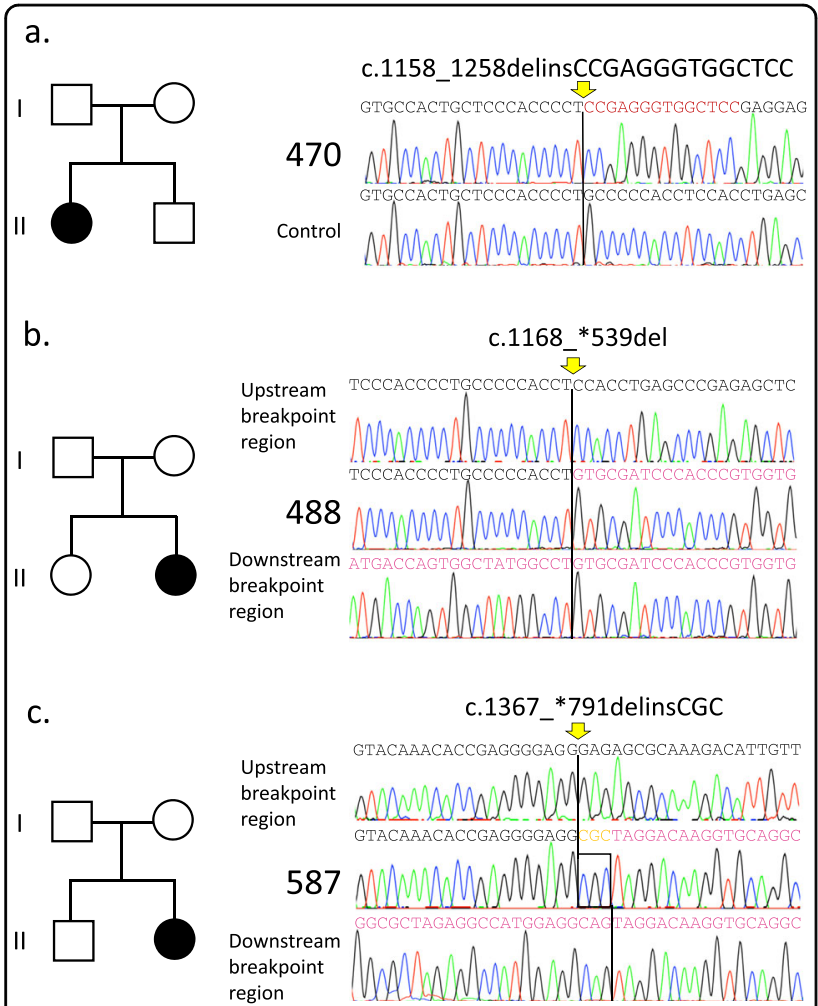

Fig. 1 Sequence analysis of the breakpoints of $M E C P 2$ structural variants in three RTT patients. a-c Pedigrees of each family (left). Nucleotide sequences of MECP2 in patient 470, 488, 587, and controls spanning the breakpoint of each structural variant. All samples were amplified by PCR and then cloned into a plasmid vector, followed by direct sequencing of the junction fragments

Patients 269, 289 and 451, no repetitive sequences were found adjacent to the breakpoint. These findings suggest that the de novo deletion events involving $M E C P 2$ can be unique to families and that homology-mediated mechanisms are unlikely to be associated with these events.

In addition, we identified known pathogenic variants in 31 patients (Supplementary Table 1). No novel change was identified, suggesting that the molecular basis for recurrent de novo nucleotide substitutions in MECP2 is common among the different populations.

The list of $M E C P 2$ variants found in 49 Japanese patients with RTT should provide a useful resource to further examine the correlation between genotypes and disease phenotypes.

\section{HGV Database}

The relevant data from this Data Report are hosted at the Human Genome Variation Database at https://doi.org/10.6084/m9.figshare.hgv.2648 https://doi.org/10.6084/m9.figshare.hgv.2651 https://doi.org/10.6084/m9.figshare.hgv.2654 https://doi.org/10.6084/m9.figshare.hgv.2657 https://doi.org/10.6084/m9.figshare.hgv.2660 https://doi.org/10.6084/m9.figshare.hgv.2663 https://doi.org/10.6084/m9.figshare.hgv.2666 https://doi.org/10.6084/m9.figshare.hgv.2669 https://doi.org/10.6084/m9.figshare.hgv.2672 https://doi.org/10.6084/m9.figshare.hgv.2675 https://doi.org/10.6084/m9.figshare.hgv.2678 https://doi.org/10.6084/m9.figshare.hgv.2681 https://doi.org/10.6084/m9.figshare.hgv.2684 https://doi.org/10.6084/m9.figshare.hgv.2687 https://doi.org/10.6084/m9.figshare.hgv.2690 https://doi.org/10.6084/m9.figshare.hgv.2693 https://doi.org/10.6084/m9.figshare.hgv.2696 https://doi.org/10.6084/m9.figshare.hgv.2699 https://doi.org/10.6084/m9.figshare.hgv.2702 https://doi.org/10.6084/m9.figshare.hgv.2705 https://doi.org/10.6084/m9.figshare.hgv.2708 https://doi.org/10.6084/m9.figshare.hgv.2711 https://doi.org/10.6084/m9.figshare.hgv.2714 https://doi.org/10.6084/m9.figshare.hgv.2717 https://doi.org/10.6084/m9.figshare.hgv.2720 https://doi.org/10.6084/m9.figshare.hgv.2723 https://doi.org/10.6084/m9.figshare.hgv.2726 https://doi.org/10.6084/m9.figshare.hgv.2729 https://doi.org/10.6084/m9.figshare.hgv.2732 https://doi.org/10.6084/m9.figshare.hgv.2735 https://doi.org/10.6084/m9.figshare.hgv.2738 https://doi.org/10.6084/m9.figshare.hgv.2741 https://doi.org/10.6084/m9.figshare.hgv.2744 https://doi.org/10.6084/m9.figshare.hgv.2747 https://doi.org/10.6084/m9.figshare.hgv.2750 https://doi.org/10.6084/m9.figshare.hgv.2753 https://doi.org/10.6084/m9.figshare.hgv.2756 https://doi.org/10.6084/m9.figshare.hgv.2759 https://doi.org/10.6084/m9.figshare.hgv.2762 https://doi.org/10.6084/m9.figshare.hgv.2765 https://doi.org/10.6084/m9.figshare.hgv.2768 https://doi.org/10.6084/m9.figshare.hgv.2771 https://doi.org/10.6084/m9.figshare.hgv.2774 https://doi.org/10.6084/m9.figshare.hgv.2777 https://doi.org/10.6084/m9.figshare.hgv.2780 https://doi.org/10.6084/m9.figshare.hgv.2783

\section{Acknowledgements}

We are very grateful to the patients who participated in this study. We thank the attending physicians and Yoko Nakamura and Yoshie Sawano (Department of Mental Retardation and Birth Defect Research, National Institute of Neurology, NCNP) for their technical contribution. This study is partially supported by the Program for an Integrated Database of Clinical and Genomic Information (17kk0205012h0002 to Y.G.) and Construction of integrated database of clinical and genomics information and sustainable system for promoting genomic medicine in Japan (18kk0205012s0303 to Y.G.) from the Japan Agency for Medical Research and Development, AMED, and the Intramural Research Grant (27-6 to Y.G.; 30-9 to A.I. for Neurological and Psychiatric Disorders of NCNP, and Joint Usage and Joint Research Programs, the Institute of Advanced Medical Sciences, Tokushima University (A.I.).

\section{Author details}

'Department of Child Neurology, National Center Hospital, National Center of Neurology and Psychiatry (NCNP), Kodaira, Tokyo 187-8551, Japan.

${ }^{2}$ Department of Clinical Genome Analysis, Medical Genome Center, NCNP, Kodaira, Tokyo 187-8551, Japan. ${ }^{3}$ Department of Mental Retardation and Birth Defect Research, National Institute of Neurology, NCNP, Kodaira, Tokyo 1878551, Japan. ${ }^{4}$ Medical Genome Center, NCNP, Kodaira, Tokyo 187-8551, Japan

Conflict of interest

The authors declare that they have no conflict of interest.

Publisher's note

Springer Nature remains neutral with regard to jurisdictional claims in published maps and institutional affiliations. 
Supplementary information is available for this paper at https://doi.org/ 10.1038/s41439-019-0078-2.

Received: 4 August 2019 Revised: 11 September 2019 Accepted: 13 September 2019.

Published online: 18 October 2019

\section{References}

1. Ip, J. P. K., Mellios, N. \& Sur, M. Rett syndrome: insights into genetic, molecular and circuit mechanisms. Nat. Rev. Neurosci. 19, 368-382 (2018).
2. Amir, R. E. et al. Rett syndrome is caused by mutations in X-linked MECP2, encoding methyl-CpG-binding protein 2. Nat. Genet. 23, 185-188 (1998).

3. Krishnaraj, R., Ho, G. \& Christodoulou, J. RettBASE: Rett syndrome database update. Hum. Mutat. 38, 922-931 (2017).

4. Hardwick, SA., Reuter, K., Williamson, SL., Vasudevan, V., Donald, J. \& Slater, K. Delineation of large deletions of the MECP2 gene in Rett syndrome patients, including a familial case with a male proband.Eur. J. Hum. Genet. 15, 1218-1229 (2007).

5. Laccone, F. et al. Large deletions of the MECP2 gene detected by gene dosage analysis in patients with Rett syndrome. Hum. Mutat. 3, 234-244 (2004).

6. Vidal, S. et al. Characterization of large deletions of the MECP2 gene in Rett syndrome patients by gene dosageanalysis. Mol. Genet. Genom. Med. 7, e793 (2019). 